\title{
Importância do Cirurgião Dentista na saúde bucal de pacientes portadores da Síndrome de Down
}

Importance of the Dental Surgeon in the oral health of patients with Down syndrome

Recebido: 29/09/2021 | Revisado: 04/10/2021 | Aceito: 08/10/2021 | Publicado: 12/10/2021

\author{
Bianca Guedes Cavalcante Lima \\ Universidade Brasil, Brasil \\ E-mail: biaguedescl@hotmail.com \\ Gustavo Gabriel Rocha de Souza \\ Universidade Brasil, Brasil \\ E-mail: gustavogrochaa@gmail.com \\ Paulo Roberto Gromatzky \\ Universidade Brasil, Brasil \\ E-mail: pgromatzky@gmail.com
}

\begin{abstract}
Resumo
A trissomia do cromossomo 21 provoca alterações físicas, motoras e psicológicas em seus portadores. Com a baixa das habilidades cognitivas e a redução da coordenação motora, os problemas orais acabam surgindo. Além disso, o número de profissionais (odontológicos e cuidadores) qualificados e/ou especializados no tratamento são baixíssimos. Por meio de revisão de literatura, este estudo aborda os detalhes relacionados à higiene bucal de pacientes com síndrome de Down, auxilia e visa melhorar a qualidade de vida desses pacientes.
\end{abstract}

Palavras-chave: Trissomia; Higiene bucal; Síndrome de Down; Odontológicos.

\begin{abstract}
Chromosome 21 trisomy causes physical, motor and psychological changes in its carriers. With the loss of cognitive abilities and reduced motor coordination, oral problems end up arising. In addition, the number of professionals (dentists and caregivers) qualified and/or specialized in the treatment is extremely low. Through a literature review, this study addresses the details related to oral hygiene of patients with Down syndrome, helps and aims to improve the quality of life of these patients.
\end{abstract}

Keywords: Trisomy; Oral hygiene; Down syndrome; Dentists.

\section{Introdução}

Pacientes portadores de necessidades especiais requerem cuidados diferenciados, sejam de longa duração ou vitalícios. Devido às restrições motoras, psicológicas ou sociais, essas pessoas costumam apresentar problemas bucais, necessitando de cuidados odontológicos especiais e procedimentos específicos. Os dentistas responsáveis devem adotar métodos de qualidade para aliviar as dificuldades. Eles tendem a ser mais sujeitos a lesões orais, como as cáries e doenças periodontais. As limitações físicas e ou mentais são um fator importante que leva ao acúmulo de placa, dificultando a higiene oral.

Os portadores apresentam diferenças genéticas causadas pela trissomia do cromossomo 21, que se separa por mitose ou não meiose, podendo ocorrer até mesmo devido a uma translocação desequilibrada desse par de cromossomos. A frequência dessa situação é de uma em mil, e a incidência pode aumentar com a faixa etária materna.

Estima-se que, no Brasil, existem poucos profissionais de saúde bucal com qualificação técnica para cuidar desses pacientes. Portanto, o acompanhamento odontológico para pacientes com síndrome de Down ainda parece desatualizado em nosso meio, o que geralmente se deve ao desconhecimento dessa condição por parte dos dentistas. Sem falar que, em alguns casos, a recusa em prestar cuidados tem um cunho discriminatório de familiares e ou profissionais de saúde por ignorância da 
sociedade. Além disso, os ajudantes nos cuidados bucais encontrem dificuldades nesse processo, pela falta de informações sobre as características e os métodos de higiene bucal adequados para esses pacientes.

O estudo se propõe a promover uma investigação sobre os cuidados bucais de pacientes com síndrome de Down. Para tanto, foi realizada uma revisão narrativa da literatura, fornecendo conhecimento sobre as características bucais desses pacientes, métodos de atendimento odontológico, métodos de tratamento, métodos de prevenção de cárie dentária, doença periodontal e higiene bucal.

\section{Metodologia}

Foi realizada uma revisão narrativa da literatura. Os materiais incluídos são as bases científicas publicadas em espanhol, inglês e português entre 1997 e 2019, e contêm os seguintes termos: "trissomia", "higiene oral", "odontologia inclusiva", "pessoas com necessidades especiais", "saúde oral" e "síndrome de Down " São usados sinônimos desses termos e seus equivalentes nessas línguas. Os veículos de dados utilizadas são Academic Google, Pubmed e Scielo.

Esta revisão de literatura inclui artigos científicos das revistas, teses e capítulos de livros listados. Como critérios de exclusão, foram considerados: materiais para os quais o texto completo não está acessível e trabalhos sem tópicos relacionados à higiene bucal de pacientes com síndrome de Down.

\section{Revisão da Literatura}

A Síndrome de Down (SD) é a “alteração genética mais comum entre os seres humanos”. (Botão et al., 2013) Caracterizada por uma má formação na distribuição dos cromossomos durante a divisão celular embrionária, revertendo para a trissomia do cromossomo 21. Sabendo que há relação da síndrome e a idade materna acima de 40 anos de idade, a porcentagem de ocorrer uma gestação de uma pessoa com Síndrome de Down é de 1\% e aos 45 é de 3\%, já aos 20 é de $0,07 \%$. (Leme \& Cruz, 2008) Essa alteração origina-se do óvulo em 95\% dos casos e do espermatozoide, em 5\% dos casos (Santos; Franceschini \& Priore, 2006).

$\mathrm{O}$ aspecto mais comum dos portadores de seus portadores é o retardo mental. Esse atraso pode ser detectado por testes de QI (Quociente de Inteligência), como leve (entre 50 e 70), moderado (entre 35 e 50) e grave (entre 20 e 35). A maioria dos pacientes apresenta leve alívio. Pacientes especiais com síndrome de Down apresentam pior desenvolvimento cognitivo e de linguagem. Esses defeitos de desenvolvimento costumam causar alguns danos aos indivíduos, como dificuldade em reconhecer regras gramaticais e fonéticas e problemas de linguagem. Essas características reduzem o vocabulário dos pacientes que por sua vez não conseguem se expressar como entendem e falam. (Alves, Silveira, \& Lins, 2007, Angélico, \& Del Pettre, 2011)

Este fato tem levado alguns estudiosos a acreditar que esses pacientes por vezes são subestimados em termos de desenvolvimento cognitivo, pois é complicado saber se eles não entendem o conteúdo transmitido a eles ou simplesmente não conseguem comunicá-lo. (Araújo, 2017, Bimstein, 2002). E o fato de possuírem características físicas típicas e comprometimento intelectual, não significa que tenham menos direitos e necessidades que outras pessoas. (Varella, 2014)

Devido às diferenças na disfunção motora e neurológica e na base óssea, os pacientes com síndrome de Down são mais suscetíveis à doença periodontal. O sistema imunológico prejudicado desses indivíduos coopera entre si para aumentar o crescimento de Actinobacillus actinomycetemcomitans, Capnocytophaga, ochracea e Porphyromonas gengivalis, que são patógenos importantes para o desenvolvimento da doença periodontal. Levando à agressividade e aos estágios iniciais da doença. (Borges, \& Diples, 2011). 
Comparando com indivíduos não sindrômicos, a doença periodontal progride mais rapidamente e de forma mais extensa, afetando a dentição decídua, o que pode levar à perda dentária prematura, bem como os dentes permanentes, em que se observa reabsorção óssea severa, mobilidade dentária e presença de cálculo dental, juntamente com o desenvolvimento de bolsas periodontais, estão relacionados ao acúmulo de biofilme dentário e inflamação gengival. (Falcão, Santos, Nascimento, Santos, \& Costa, 2019). Causam uma inflamação generalizada, predominantemente mais severa em dentes inferiores. (Borges, \& Diples, 2011).

Eles podem ter problemas cardíacos, respiratórios e disfunção da tireoide (portanto, são propensas ao excesso de peso. Essas pessoas costumam ser muito dóceis, cooperativas, bem-humoradas e alegres (Marra, 2007). Araújo (2017) acrescenta que, além de ouvir música, assistir TV, folhear e colorir desenhos como passatempo e entretenimento, más também possuem as características de "raiva", excitação ou irritabilidade. Esses pacientes também podem sofrer de transtorno de ansiedade crônica, que leva a um controle neurológico inadequado. Mendonça, Pfeifer, \& Nascimento, 2012) Essa ansiedade crônica pode levar a um aumento significativo na incidência de bruxismo, desgastando os sulcos e as fissuras dos dentes, tornando-os de espessura lisa, provocando assim fraturas dentárias devido à sobrecarga dos tecidos de suporte. (Oliveira, Ramos, \& Paiva, 2001)

Devido à deficiência física, motora e intelectual, esses pacientes costumam ter dificuldade no uso da escova durante a escovação e ao utilizar o fio dental nos cuidados pré-escovação, onde acumula biofilme e favorece o aparecimento de doenças bucais. (Oliveira, Ramos, \& Paiva, 2001)

A qualidade de vida dessas pessoas pode depender em grande parte da família, principalmente nos estágios iniciais, quando ocorre grande parte do desenvolvimento cognitivo, motor e emocional. Portanto, nas primeiras fases da vida, da infância à adolescência, é muito importante a convivência com os familiares, pois uma série de dificuldades surgirão nesse período, sendo os pais e demais familiares imprescindíveis no acompanhamento dessas mudanças. (Oliveira, Luz, \& Paiva, 2008)

Pacientes com síndrome de Down apresentam diversas características clínicas e fenotípicas, tais como: flexibilidade articular excessiva, baixa estatura, membros encurtados (mãos, pés, dedos, orelhas e nariz), pescoço curto e largo, pés curtos, pés largos e grossos, o arco projeta-se entre o primeiro e o segundo dedo do pé, e a orofaringe e a nasofaringe são estreitas e reduzidas. Além disso, há faces redondas (branquicefálica), orelhas baixas e palato estreitos e profundo. (Santangelo, Gomes, Vilela, Deus, Vilela, \& Santos, 2008)

Esses pacientes também apresentam algumas alterações orais, características de seu quadro clínico. As principais características orais da síndrome são: dificuldade para respirar pela boca, macroglossia, fenda de língua, hipotonia, palato alto,classe III de Angle, menos cárie, dentes posteriores, abertura e fechamento, e doença periodontal grave. Quanto às mudanças dentárias que existem na síndrome de Down, as mais comuns são: dentes pequenos, dentes em forma de cone, brotamento, dentes decíduos e permanentes, dentes perdidos, hipocalcificação do esmalte, fusão e erupção dentária retardada. (Silva, Neto, \& Pires, 1997)

Em comparação com pacientes não sindrômicos, os pacientes com síndrome de Down apresentam menor prevalência de cárie dentária. Isso se deve ao aumento do pH 15 concentrado na saliva dessas pessoas.

A língua fissurada é um pequeno sulco que aparece na superfície da língua, tornando-a mais profunda e, portanto, propícia para a retenção de alimentos. São rastros indolores, de forma que o acúmulo de alimentos passa despercebido. Esse acúmulo pode levar ao mau hálito, também denominado de "mau hálito" nos indivíduos. (Teitelbaum, \& Sabbagh-Haddad, 2007)

Sendo uma doença genética rara, a macroglossia que ocorre mais comumente em crianças e pode causar alguns problemas funcionais relacionados à dentição. Isso se deve ao crescimento excessivo do tecido muscular da língua, levando à 
sua hipertrofia, o que pode causar muitos problemas ao indivíduo. Ainda há muita discussão sobre o tratamento dessa condição. (Teitelbaum, \& Sabbagh-Haddad, 2007)

Devido à má higiene oral, alveolar pequeno, raiz de dente curta e certa hipoplasia dentária, a Periodontite em pacientes com síndrome de Down, A taxa de aparecimento de periodontite é bastante rápida, especialmente na faixa etária após os 30 anos de idade. (Van de Wiel, Van Loon, Reuland, \& Bruers, 2018)

\section{Discussão}

Entendemos que, para pacientes com síndrome de Down, o cirurgião dentista adequado deve monitorar desde o nascimento até o envelhecimento, a fim de fornecer atendimento humano e multidisciplinar a cada paciente com essa síndrome. Para melhorar a saúde desses pacientes especiais, os profissionais utilizam todo o seu conhecimento como método de tratamento, com o auxílio de maquetes macroscópicas, desenhos coloridos e brinquedos, que ajudam o paciente a entender o que será feito com ele. O papel do paciente no tratamento é fundamental para direcionar sua atenção e estimular a necessidade de prevenção de doenças bucais. A família também é uma inspiração muito importante para a realização dos procedimentos, pois será ela a responsável por dar continuidade ao tratamento em casa para proporcionar saúde bucal adequada ao portador da síndrome de Down.

\section{Considerações Finais}

A síndrome de Down costuma ter características que afetam negativamente o comportamento de higiene oral, como déficits cognitivos e diminuição da coordenação motora. Além disso, geralmente apresentam algumas doenças bucais específicas, como fissuras, hipertrofia da língua e dentes cônicos. Devido às dificuldades em fornecer higiene bucal a esses pacientes, o principal motivo é que o número de profissionais capacitados é pequeno e o pessoal responsável pela atenção domiciliar carece de informações. A partir da literatura revisada, e levando em consideração a relevância da inclusão social para pacientes com necessidades especiais, destaca-se a importância do dentista em considerar as características físicas e psicológicas inerentes aos pacientes com síndrome de Down para promover cuidados não invasivos e mais frutíferos.

Mais propensos a características orais específicas, como macroglossia, hipotonia, palato alto, alterações dentais significativas usados por profissionais da odontologia. Os seguintes comportamentos são usados com cautela como um método de cuidado. Inclui acompanhamento familiar e prevenção. Com relação às operações realizadas em casa por cuidadores e responsáveis de pacientes com síndrome de Down, recomenda-se que procurem o aconselhamento de um dentista e estudem os melhores métodos de síndrome de Down e higiene bucal.

Vale ressaltar a importância dessas pessoas na promoção da saúde bucal dos pacientes com síndrome, não só para auxiliá-los nesse processo, mas também para motivá-los a realizar a higiene bucal. De forma positiva, confirmando-se que preconceito e discriminação são inimigos da inclusão social.

\section{Referências}

Alves, R.D., Silveira, E.J.D \& Lins, R.D.A.U. (2007). Doença Periodontal X Síndrome de Down: Uma Revisão. Recuperado de: www.patologiaoral.com.br/texto63.asp.

Angélico, A. P. \& Del Pettre, A. (2011). Avaliação do repertório de habilidade sociais de adolescentes com Síndrome de Down. Psicologia Reflexão e Crítica. Araújo, S. C. V. (2017). Adolescência, Síndrome de Down e sintomas psicóticos a partir da perspectiva winnicottiana: Relato de um caso. Brasília, UniCEUB/ICPD.

Bimstein, E. (2002). et al. Saúde e doenças periodontais e gengivais: crianças, adolescentes e adultos jovens. São Paulo, Santos. 
Borges, F. T. M. \& Diples, A. B. (2011). Síndrome de Down: Relação Pais, Filhos e Sociabilidade. Anuário da Produção Acadêmica Docente. São João da Boa Vista, v. 5.

Botão et al., (2013), p. 2375.

Coelho, C. (2016). Síndrome de Down.

Falcão, A. C. S. L. A., Santos, J. M., Nascimento, K. L. L., Santos, D. B. N. \& Costa, P. V. A. (2019). Síndrome de Down: abordagem odontopediátrica na fase oral. Revista Odontol. São Paulo, v. 31

Gomes, J. I. R. \& Ribeiro, S. M. S. (2019). Condições periodontais de pacientes portadores de Síndrome de Down. Porto Velho, Centro Universitário São Lucas, 2019. 17 f. Dissertação - Programa de Graduação em Bacharel em Odontologia, Centro Universitário São Lucas, Porto Velho.

Leme \& Cruz, (2008).

Marra, P. S. (2007). Dificuldades encontradas pelos responsáveis, para manter a saúde bucal em portadores de necessidades especiais. Duque de Caxias, Universidade do Grande Rio "Prof. José de Souza Herde", 2007. 108 f. Dissertação (mestrado) - Programa de mestrado em Odontologia, Universidade do Grande Rio "Prof. José de Souza Herde", Duque de Caxias.

Mendonça, L. F. R., Pfeifer, L. I., Sigolo, S. R.R. L. S. \& Nascimento, L. C. (2012). Inclusão de crianças com Síndrome de Down. Psicol. Estud.

Oliveira, A.C.B., Ramos, J.M.L. \& Paiva, S.M. (2001). Aspectos relevantes à abordagem odontológica da criança com síndrome de Down. Revista do CROMG.

Oliveira, A.C., Luz, C. L.F. \& Paiva, S. M. (2008). O papel da saúde bucal na qualidade de vida do indivíduo com Síndrome de Down. Arquivos em Odontologia.

Santangelo, C. N., Gomes, D. P., Vilela, L. O., Deus, T. S., Vilela, V. O. \& Santos, E. M. (2008). Avaliação das características buscais de pacientes portadores de Síndrome de Down da APAE de Mogi das Cruzes - SP. ConScietiae Saúde.

Silva, F.A., Neto, J.V. \& Pires, C.C.C. (1997). Síndrome de Down: peculiaridades de interesse odontológico e possibilidades ortodônticas. Rev. Fac Odontol $U F G O$.

Santos, Franceschini \& Priore, (2006).

Teitelbaum, A.P. \& Sabbagh-Haddad, A. (2007). Alteração sistêmica na Síndrome de Down e cuidados no atendimento odontológico. Rev Assoc Paul Dent.

Van de Wiel, B., Van Loon, M., Reuland, W. \& Bruers, J. (2018). Periodontol disease in Down's syndrome patients. A restrospective study. Spec Care Dentist. 